\title{
ON THE PROJECTIVE AXIOMS OF GEOMETRY*
}

\author{
BY
}

\section{ELIAKIM HASTINGS MOORE}

\section{Introduction.}

In the present paper I wish to consider the axioms called by Hilbert $\dagger$ (1899) the axioms of connection and of order (I 1-7, II 1-5 of HILBERT's list), and called by ScHUR $\ddagger$ (1901) the projective axioms of geometry.

Hilbert states (l. c., pp. 3,21 ) that his body of axioms consists of independent axioms, that is, that no one of the axioms is logically deducible from the remaining axioms. This statement, accepted by HöLDER $\S$ and Sommer, $\|$ is not accepted by Schur, $\uparrow$ who holds that HilberT's axioms I 3, 4, 5 are consequences of the axioms I 1-2, 7, II 1-5. For reasons explained below, this last remark cannot be accepted as valid.

There are, however, redundancies ** in HILBERT's axioms I-II, the two axioms

* Presented to the Society December 28, 1901. Received for publication December 31, 1901.

† Hilbert, Grundlagen der Geometrie, Festschrift zur Feier der Enthüllung des Gaus8Weber-Denkmals in Göttingen, Leipzig, 1899.

† Schur, Ueber die Grundlagen der Goometrie, Mathematische Annalen, vol. 55 (1901), pp. 265-292.

§ Hölder, Anschauung und Denken in der Geometrie, Leipzig, 1900 ; cf. p. 35.

|| Sоммек, Hilbert's Foundations of Geometry, Bulletin, vol. 6 (1900), p. 292.

Tloc. cit., pp. $266,267,271$.

** As to the independence of the axioms I 1-7, II 1-5 HILnERT (l. c., p. 21) refers to his Göttingen lectures of the winter semester 1898-99, as reported by DR. voN SCH APER and manifolded. By the kindness of Dr. Bosworth-Focke I have seen a copy of this report. Proofs are given that (1) I 2 is independent of I 1 ; (2) I 5 of I 1-4, 6-7; (3) I 6 of I 1-5, 7 ; (4) II 4 of I 1-7, II 1-3; (5) II 3 of I 1-7, II 1-2, 4 ; (6) II 5 of I 1-7, II 1-4.-In contravention of the remark of SCHUR, I prove below further that (7) I 3 is independent of I 1-2, 4-7, II 1-5 ; (8) I 4 of I 1-2, 6-7, II 1-5; (9) I 5 of I 1-3, 6-7, II 1-5.

Each of these proofs involves the exhibition of a particular geometry, in which, e. g., in case (9) the relations I 1-3, 6-7, II 1-5 hold while the relation I 5 does not hold. This exhibition is in terms of a science supposed to exist without self-contradictions, and the proposition of independence is contingent on the correctness of this supposition. As science of reference one prefers the usual analysis of real numbers or a science (e. g., the analysis of complex numbers; euclidean $n$-dimensional geometry) whose existence is deducible from that of analysis.

Such proofs were used by PEANo in investigations on the foundations of arithmetio and geometry (1. c., p. 62) and they play a brilliant part in the work of HILBERT. 
I 4 , II 4 being deducible from I $1-3,5,7$, II 1-3, 5. I establish these two conclusions in $\S 5$ of this paper.*

In $\S 1$ I give a simplified arrangement of the projective axioms of $n$-dimensional geometry $(n \geqq 2)$. I use as elements the point and the line and the segment of a line, the line and the segment being certain sets of points, and give convenient definitions of the plane or 2 -space, and the $k$-spaces in general $(0 \leqq k \leqq n)$; the properties of the $k$-spaces are developed in $\S 3$.

It is convenient here briefly to characterize the systems of projective axioms of 3-dimensional geometry given by $\mathrm{PASCH} \dagger(1882)$, Peano $\ddagger(1889,1894)$. Ingrami § (1899) and Hilbert (1899).

PASCH undertook to make pure geometry in a strict sense a purely deductive (abstract) science based on certain assumed (abstract) notions subject to certain assumed relations. He introduced as basal notions for geometry the point, the linear-segment (geráde Strecke) and the planar-segment (ebene Fläche), in terms of which the line and the plane were defined. The linear-segment and the planarsegment are certain sets of points.

Peano, following Pasch, retained the point and the linear-segment; but he defined the line and the plane by means of these elements alone. His system, as simplified by Ingrami, is given by Schur (l. c., p. $267 \mathrm{ff}$.)

Hilbert has as basal notions the point, the line, the segment of a line (connected with the notion between) and the plane. The line and the plane are by the Hilbert axioms (I 1, 7 ; I 3, $7_{2}$ ) connected with certain sets of points (lying on the line; the plane), in such a way that we may (and for simplicity we do) identify them with those sets of points. For convenience of reference I set down the Hilbert axioms I 1-7, II 5 ; the axioms II 1, 2, 3 are in effect the axioms $2,5,3$ of $\S 1$ of this paper, while axiom II 4 is quoted in the theorem of $\S 2$.

I 1, 2, $7_{1}$. Two distinct points $A, B$ determine in every case a line $g$; we set $A B=g$ or $B A=g$. Any two distinct points of a line determine this line; that is, if $A B=g$ and $A C=g$, and $B \neq C$, then $B C=g$. On every line there are at least two points.

I $3,4,5,7_{2}$. Three non-collinear points $A, B, C$ determine in every case $a$ plane $\pi$; we set $A B C=\pi$. Any three non-collinear points of a plane determine this plane. If two points $A, B$ of a line $g$ lie in a plane $\pi$, then every point of the line $g$ lies in $\pi$. On every plane there are at least three non-collinear points.

* This paper has been prepared in connection with my current Chicago seminar-course on the foundations of geometry and analysis, and queries and remarks of members of this course, in particular, of Mr. O. VeBLEN, have been a source of much stimulus.

† РАSCH, Voriesungen über neuere Geometrie, Leipzig, 1882.

$\ddagger$ Peano, Iprincipii di Geometria, Torino, 1889 (cited in the following paper), Sui fondamenti della Geometria, Rivista di Matematica, vol. 4 (1894), pp. 51-90.

§INGrami, Elementi di Geometria per le scuole secondarie superiori, Bologna, 1899 (oitation of SCHUR). 
I $7_{3}, 6$. There exist at least [one plane and] * four non-coplanar points. If two planes $a, \beta$ have a point $A$ in common, then they have at least a second point $B$ in common.

II 5. Let $A, B, C$ be three non-collinear points and $g$ a line lying in the plane $A B C$ and containing no one of the points $A, B, C$; if the line $g$ contains a point within the segment $A B$, then it always contains either a point within the segment $A C$ or a point within the segment $B C$.

Here every one of two (three) points which determine a line (plane) is defined as a point of or on or in or lying in the line (plane).- In interpretation we agree: (1) I 2 has the full meaning of its original statement, viz.: If $A B=g$ and $C D=g$, and $B \neq D$, then $B D=g . \cdot(2)$ I 4 has the specification: If $A_{1} A_{2} A=\pi, B_{1} B_{2} B=\pi$ and $C_{1} C_{2} C=\pi$, and $A, B, C$ are non-collinear, then $A B C=\pi$. (3) In I 3 , if $A B C=\pi$, then $B A C=\pi$ and $A C B=\pi$. (4) The determinations in I 1, 3 are unique determinations.

Clearly the body of axioms of a system depends essentially upon the choice of the basal notions of the system. In this connection a remark is pertinent with respect to one's attitude concerning the foundations of geometry. I suppose that if geometry $\dagger$ is taken to be a natural science-the science or a science of the space in which or according to which we live-it would, as is contended by $\mathrm{PASCH}$ and Peano, be undesirable to introduce the line as a basal notion. The linear-segment seems to be a more fundamental notion. But we may discriminate between that part of geometry which establishes a body of postulates based as directly as may be on spatial experience or intuition, and that part which consists in the organization of the science on the basis of the accepted body of axioms; and so we understand that it may in the development of the theory be convenient to replace the body of primary notions and relations by another body of notions and relations, less fundamental, but, with respect to the deductive geometry, more convenient. I suppose that with this thought Hilbert introduced the line and the plane as basal notions in his abstract geometry.-It is understood that greater generality would be obtained by introducing the axioms as valid, as one says, for a limited region of space. In this connection reference is made to KLEIN's introduction $\ddagger$ of the ideal elements of projective geometry without the use of the parallel axiom, and to the remarks of PASCH (pp. 4, 18, 126), of Peano (p. 75) and of Schur (pp. 267, 274).

As has been stated, ScHUR has remarked that in the body of projective axioms of Hilber'T the axioms I 3, 4, 5 are redundant. This criticism is, however,

* The bracketed addition to the original form of $\mathrm{I} 7_{3}$ is a necessary addition; it is an implication of the remarks with which HILBERT introduces the axioms I.

† For $n=3$. - In case $n>3$ the geometry is perhaps essentially abstract.

$\ddagger$ Mathematische Annalen, vol. 6, p. 134.-Cf. ScHuR, ibid., vol. 39, p. 113, and vol. 55 , p. 274 . 
incorrect. For if in the ordinary euclidean 3-dimensional space we take the points, the lines, the segments of lines, and two intersecting spheres as the points, the lines, the segments of lines, and the (only) planes of a new geometry one has a geometry in which I 1-2, 6-7, II 1-5 are satisfied, while all the statements I $3,4,5$ are invalid. Thus $I 3,4,5$ are not logical consequences of the remaining axioms I, II. Indeed, one may prove $*$ that (1) I 3 is independent of I $1-2,4-7$, II $1-5$; (2) I 5 of I $1-3,6-7$, II $1-5$. These two remarks together with the fact (already stated) that I 4 is deducible from I 1-3, 5, 7, II 1-3, 5 furnish a satisfactory account of the rôles of the axioms I $3,4,5$ in the Hilbert system I, II.

The remark of Schur was connected with the fact that, as Peano pointed out, the plane is capable of explicit definition. Apparently ScHur directly identified the Hilbert plane with the Peano plane. But in a particular Hilbert geometry the plane postulated by HILBERT is to be identified with the plane defined by Peano certainly only by the mediation (to some extent) of the axioms I $3-7$, II 5 by which alone the Hilbert plane is conditioned. Pursuing this thought I find $(\S 5)$ that by replacing in the Hilbert system axioms I $3,5,7$, II 5 by axioms considerably milder one is still able to make this identification and thus to prove the axioms in their original form, as used by HILBERT.

Evidently, with Peano and Schur, we may better define the plane explicitly than thus, by the mediation of axioms, implicitly. The implicit definitions of the $k$-spaces in $n$-dimensional geometry (cf. Hilbert, loc. cit., p. 71) would be still more undesirable. The explicit definitions of $\S 1$ seem to be simpler than those of $\S 4$, the analogues of the Peano-Ingrami definitions.

To indicate briefly the arrangement of this paper :

In $\S 1$ I formulate a body of seven projective axioms of $n$-dimensional geometry $(n \geqq 2)$; these for $n=3$ correspond with HILBERT's axioms I, II as follows :

$$
(1 ; 2 ; 3 ; \underline{4} ; 5 ; \underline{6} ; \underline{7})=\left(\mathrm{I} 1,2,7_{1} ; \mathrm{II} 1 ; \mathrm{II} 3 ; \underline{\mathrm{II}} 5 ; \mathrm{II} 2 ; \underline{\mathrm{I}_{2}, 7_{3}} ; \underline{\mathrm{I}} \mathbf{6}\right) ;
$$

the axioms I $3,4,5$ are omitted, the plane (and the $k$-spaces, in general) being explicitly defined; in the cases underscored the correspondence is only approximate; in all cases the effort has been to give clear and precise expression to the meaning intended to be conveyed.-Axicm 4, the triangle-transversal axiom, is funda-

* A geometry for case (1) is the ordinary euclidean 3-dimensional geometry, with the omission of one plane ; one for case $(2)$ is the ordinary euclidean 2 -dimensional geometry with the modification that the general plane $A B C$ is the set of points, $O$ apart, of the lines $A O, B O, C O$, the point $O$ being the center of the cirole inscribed in the triangle $A B C$.

One has the feeling that, even on the assumption that the Hilbert plane $A B C$ is defined explicitly as identical with the Peano plane $A B C$, the criticism of ScHUR remains incorrect, in view of the fact that the plane order axiom II 5 relates to a line supposed to lie in the plane and is accordingly in this respect milder than PFA No's two triangle axioms fundamental in the theory of the Peano plane. A geometry showing the oorrectness of this conjecture $I$ have not found.

Trans. Am. Math. Soc. 10. 
mental in the theory of the $k$-spaces; it replaces (its two corollaries 3,4 ) the two triangle axioms introduced by Peano as fundamental for the theory of the plane, which replaced PASCH's plane order axiom, retained by HILBERT as axiom II 5. -Axiom 7, the axiom of limitation, in agreement with ScHUR (loc. cit., p. 271), I think desirably replaces HILBERT's I 6.

HilberT's axiom II 4 is in $\S 2$ proved as a theorem depending on axioms 1-6, in particular on axiom 4, the triangle-transversal axiom.

In $\S 3$ the properties of the $k$-spaces are developed on the basis of the definitions of $\S 1$ and the axioms 1-6. Comparison with another type of definition is made in $\S 4$.

In $\S 5$ I prove that in Hilbert's system with the body of axioms I 1-3, 5, 7, II 1-3, 5, or with the milder body of axioms I 1, 2, 3', $5^{\prime}, 7_{1}, 7_{2}^{\prime}, 7_{3}^{\prime}$, II 1-3, $5^{\prime}$ one may identify the Hilbert plane with the plane defined in $\S 1$, and that the axioms 1-6 $(n=3)$ of $\S 1$ hold. Then it follows (by $\S \S 2,3,4)$ that the Hilbert axioms I 4, II 4 are deducible from the Hilbert axioms I 1-3, 5, 7, II 1-3, 5 .

\section{$\S 1$. The projective axioms of $n$-dimensional geometry.}

In an abstract deductive geometry (of space of $n$ dimensions, $n \geqq 2$ ) we consider a set of things called points, having properties fully* determined by the body of postulates or axioms laid down as the basis of the geometry. The space under consideration, call it the fundamental space, is the set of all points under consideration.

The projective axioms involve certain sets of points, called lines, and certain sets of collinear points, called segments (of lines). These lines and segments receive definition only implicitly by the mediation of the axioms.

The projective axioms may be grouped as follows :

1-4: Axioms of conditioned existence and of definition;

5-6 : Axioms of absolute existence;

7 : Axiom of limitation.

The reader will understand that the designations attached to the various groups of axioms are intended to indicate somewhat clearly, although roughly, what seem to be the principal functions of the various axioms in the body of axioms.

Aхгом 1: The Line. Two distinct points $A, B$ determine uniquely $a$ set of points, the line $A B$. The points $A, B$ belong to or lie on the line $A B$. A line is a set of points thus determined by certain two and indeed by any $\dagger$ two distinct points belonging to it.

* In so far as they pertain to the arbitrary geometry of the system of geometries in question.

$\dagger$ This statement may be replaced by these three: The line $A B$ contains and thus is identical with the line $B A$. The line $A B$ contains the line $A C$, where $C$ is any point not $A$ of the iine $A B$. The line $A C$ contains the point $B$. 
Axiom 2: The Segment of a Line. Two distinct points $A, B$ of a line determine uniquely a set of points distinct from $A, B$ and lying on the line; this set of points is the segment $A B$, with extremities $A, B$. The segment $A B$ contains and thus is identical with the segment $B A . A$ segment is a set of points thus determined by two distinct points, its extremities.

Def. 1. A point $C$ of the segment $A B$ is said to lie between or to separate* the extremities $A, B$ of the segment, in notation $A C B$ or $B C A$.The notation indicates the order-relation of the three points.

Axiom 3: Three Collinear Points. Of three distinct collinear points $A, B, C$ one and only one lies on the segment determined by the other two: of the three order-relations, $A B C, B C A, C A B$ one and but one $\dagger$ holds.

Axiom 4: The Triangle* with Transversal Line. A line which cuts $\ddagger$ one side of a triangle externally and another side internally cuts the thivd side internally.

In other words (cf. fig. 1):

Aхгом 4. If $A, B, C$ are three non-collinear points and if the line ${ }_{D E}^{D F}$ cuts the lines ${ }_{B C}^{B C},{ }_{C A}^{A B}$ in points ${ }_{D}^{D},{ }_{E}^{F}$ respectively, where ${ }_{B C D}^{B C D}$ and ${ }_{C E A}^{A F B}$, then it cuts the line ${ }_{A B}^{C A}$ in a point ${ }_{F}^{E}$ such that ${ }_{A F B}^{C E A}$.

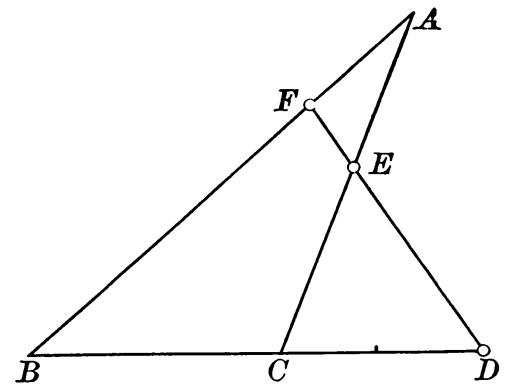

Fig. 1.

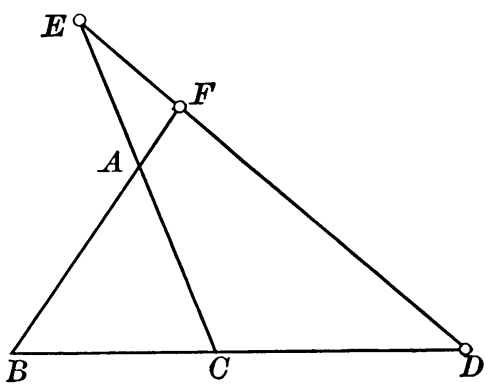

FIG. 2.

Notation. We denote the axioms into which axiom 4 has just been analyzed by the notations§

$$
\begin{array}{ll}
4_{1} & \text { or }
\end{array} \quad 4_{11}, \frac{4_{12}}{4_{2}}
$$

\footnotetext{
* Similarly, a set $\Gamma$ of pointe having a point $C$ in common with the segment $A B$ is said to separate $A$ and $B$.

$\dagger$ Here, as Mr. VeBLEN has remarked, it is obviously sufficient to state that the relation $A C B$ does not hold if the relation $A B C$ does hold.

$\ddagger$ For brevity I use, without explanation, a few geometric terms whose meaning in the abstract geometry in question is supposed to be sufficiently clear.

§Similar self-explanatory partitional suffix-notations will be used elsewhere in this paper.
} 
Remark. Axiom $4_{22}$ is a logical consequence* of axioms $4_{11}, 4_{12}$ and $4_{21}$ together with axioms $1-3$.

Remark. The following seven theorems depend so immediately on axiom 4 by the mediation of the preceding axioms that $I$ insert them here as corollaries of axiom 4.

Cor. 1. (In the notation of the second statement of axiom 4.)-The three points $D, E, F$ are distinct and such that $D E F$. The intermediate point $E$ lies on the side adjacent to the point $D$ lying on its side externally.-One applies axiom $4_{1}$ to the transversal $A C$ of the triangle $B D F$.

Cor. 2. If a line cuts two sides of a triangle externally and (cyclically) similarly, then it cuts the third side externally but dissimilarly, and the point of intersection with the third side lies between the points of intersection with the other two sides.-That is (cf. fig. 2), in analogous notation: If ${ }_{B C D}^{B C D}$ and ${ }_{A B F}^{C A E}$, then ${ }_{A C E}^{B A F}$ and ${ }_{D E F}^{D F E}$.-One applies axiom $4_{2}$ and cor. 1 to the transversal ${ }_{A C}^{B A}$ of the triangle ${ }_{B D F}^{C D E}$. - In this case the two statements are equivalent.

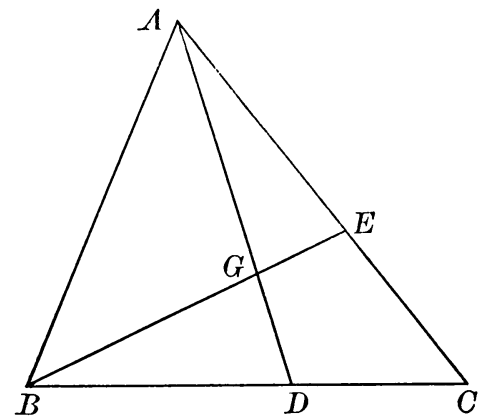

Fig. 3.

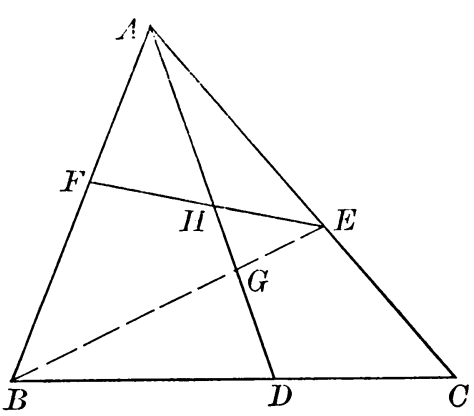

Fig. 4.

Cor. 3. The two segments $A D, B E$ from two vertices $A, B$ of a triangle $A B C$ to two points $D, E$ of the respectively opposite segments $B C, C A$ have in common a point $G$.-One applies axiom $4_{1}$ and cor. 1 to the transversal $A D$ of the triangle $B C E$. Cf. fig. 3 .

Cor. 4. The point $G$ being any point of the segment $A D$ from the vertex $A$ of a triangle $A B C$ to a point $D$ of the opposite segment $B C$, there exists a

* By use of axioms $4_{21}$ and 3 we see that it is sufficient to prove that the relations $A B F^{3}$ and $B A F^{\prime}$ are impossible, and here we use besides axiom $4_{1}$ itself its corollary 7 (of the text) which is independent of axiom $4_{2}$.

The relation $A B F^{\prime}$ is impossible. For by $4_{1}$ the transversal $F^{\prime} E$ of the triangle $A B C$ would yield the relation $B D C$.

Further, the relation $B A F$ is impossible. For, in conjunction with $D E F^{\prime}$, it would imply that the line $A E C$ meets the triangle $B D F$ in inner points of its three sides, which by cor. 7 is impossible; in conjunction with $D F E$, it would imply, by $4_{1}$ for the triangle $C E D$ with the transversal $B A H^{\prime}$, the relation $C^{\prime} A E^{\prime}$; and similarly, in conjunction with $E D F$, it would lead to the relation $A C E$. 
point $E$ of the segment $C A$ such that the segment $B E$ contains the point $G$. -One applies axiom $4_{2}$ and cor. 1 to the transversal $B G$ of the triangle $A C D$. Cor. 5. In a triangle $A B C$ the line $A D$, containing the vertex $A$ and $a$ point $D$ of the opposite segment $B C$, and the segment $E F$, from a point $E$ of the segment $C A$ to a point $F$ of the segment $A B$, have in common a point* $H$.-One applies axiom $4_{1}$ to the line $A D$ as a transversal first of the triangle $B C E$ and then of the triangle $B E F$. Cf. fig. 4 .

Cor. 6. In a triangle $A B C$ the line $A H$, containing the vertex $A$ and $a$ point $H$ of the segment $E F$, from a point $E$ of the segment $C A$ to a point $F$ of the segment $A B$, contains a point $D$ of the segment $B C$.- One applies axiom $4_{2}$ to the line $A H$ as a transversal first of the triangle $B E F$ and then of the triangle $B C E$.

Cor. 7. No transversal meets the three sides of a triangle internally.-If three points $D, E, F$ on the sides $B C, C A, A B$ of the triangle $A B C$ were collinear, then, for instance, $E$ would lie between $D$ and $F$, and further, however, by cor. 5 , the line $A D$ would meet the segment $E F$ in a point-in fact, the point $D$-lying between $E$ and $F$.

Def. $2(k=2)$. Three distinct points $A_{1}, A_{2}, B_{0}$ are (in the order specified) independent if $B_{0}$ does not lie in the line $A_{1} A_{2}$.

Def. $3(k=2)$. Three independent points $A_{1}, A_{2}, B_{0}$ determine uniquely a plane, or 2-space, $A_{1} A_{2} B_{0}$. The plane $A_{1} A_{2} B_{0}$ is the aggregate set of all points $A$ of the line $A_{1} A_{2}$, of all points $C$ separated (cf. def. 1) from $B_{0}$ by the line $A_{1} A_{2}$, and of all points $B$ separated from at least one of these points $C$ by the line $A_{1} A_{2}$.

Def. $2(k=3)$. Four points $A_{1}, A_{2}, A_{3}, B_{0}$ are independent if $A_{1}, A_{2}, A_{3}$ are independent and $B_{0}$ does not lie in the plane $A_{1} A_{2} A_{3}$.

Def. $3(k=3)$. Four independent points $A_{1}, A_{2}, A_{3}, B_{0}$ determine uniquely a 3-space $A_{1} A_{2} A_{3} B_{0}$. The 3 -space $A_{1} A_{2} A_{3} B_{0}$ is the aggregate set of all points $A$ of the 2 -space $A_{1} A_{2} A_{3}$, of all points $C$ separated from $B_{0}$ by the 2-space $A_{1} A_{2} A_{3}$, and of all points $B$ separated from at least one of these points $C$ by the 2 -space $A_{1} A_{2} A_{3}$.

Def. $2(k=k)$. The $k+1$ points $A_{1}, \cdots, A_{k}, B_{0}$ are independent if the first $k$ points $A_{1}, \ldots, A_{k}$ are independent and the last point $B_{0}$ does not lie in the $(k-1)$-space $A_{1} \cdots A_{k}$.

Def. $3(k=k) \cdot \dagger \mathrm{A}$ set of $k+1$ independent points $A_{1}, \ldots, A_{k}, B_{0}$ de-

* It is to be noted that on the basis of axioms 1-5, the point $H$ lies not only on the segment $E F$ but also on the segment $A D$. - Denoting by $G$ the point of intersection of the line $A D$ and the segment $B E$, we have, by cor. 1, $A G D$ and $A H G$, from which, by the theory of the orderrelations of four collinear points $(\$ 2)$ we have indeed $A I I D$.

$\dagger$ Denoting a point as a 0 -space, we define similarly the notions: 'Two independent points $A_{1}, B_{0}$; the 1-space $A_{1} B_{0}$. Then within a fundamental space, which contains a 2 -space, by the theory of $\S 2$, the 1-space $A_{1} B_{0}$ and the line $A_{1} B_{0}$ are identical. This remark makes general, for positive integral values of $k$, the notions of $k+1$ independent points and of a $k$-space.-Evidently it does not involve an explicit definition of the notion line. 
termines uniquely a $k$-space $A_{1} \cdots A_{k} B_{0}$; the $k$-space $A_{1} \cdots A_{k} B_{0}$ is the aggregate set of all points $A$ of the $(k-1)$-space $A_{1} \cdots A_{k}$, of all points $C$ separated from $B_{0}$ by the $(k-1)$-space $A_{1} \cdots A_{k}$, and of all points $B$ separated from at least one of these points $C$ by the $(k-1)$-space $A_{1} \cdots A_{k}$.

Aхгом 5. On the line $A B$ determined by two distinct points $A, B$ there exists a point $C$ on the segment $A B$ and there exists a point $D$ distinct from $A$ such that the segment $A D$ contains $B$.

Aхгом 6. There exists in the fundamental space a set of $n+1$ independent points $A_{1}, \cdots, A_{n}, A_{n+1}$ (where $n$ is any particular integer greater than 1 ); and thus there exists $a$ set of $k+1$ independent points $A_{1}, \cdots, A_{k}, A_{k+1}$ for every integer $k(1 \leqq k \leqq n)$.

Cor. The fundamental space contains $k$-spaces $(1 \leqq k \leqq n)$.

Aхгом 7. There exists in the fundamental space* no $(n+1)$-space.

Cor. The fundamental space contains no set of $n+2$ independent points. In the system of axioms, axioms 6 and 7 may be condensed into the following Axiom. The fundamental space is an n-space.

§ 2. The order-relations of four collinear points. Deduction from axioms $1-4,5_{1}, 6(n \geqq 2)$.

In the usual geometry one will admit the validity of the following four statements concerning the order-relations of four distinct collinear points $A, B$, $C, D$.

1. If $C A B$ and $A B D$, then $C A D$ and $C B D$.

2. If $C A B$ and $A D B$, then $C A D$ and $C D B$.

3. If $C A B$ and $D A B$, then either $C D A$ or $D C A$; either $C D B$ or $D C B$; if $C D A$, then $C D B$; and if $C D B$, then $C D A$.

4. If $A C B$ and $A D B$, then either $A C D$ or $A D C$; either $C D B$ or $D C B$; if $A C D$, then $C D B$; and if $C D B$, then $A C D$.

Concerning these four statements we establish the fundamental

Theorem. The statements $1,2,3,4$ are evidently equivalent to Hilbert's linear order axiom II 4:

To any four distinct points of a line the notation $A, \cdot B, C, D$ may always be assigned in such a way that $A B C, A B D, A C D, B C D$. Furthermore, these statements are theorems of a geometry in which axioms $1-4,5$ and 6 for $n \geqq 2$ hold.

We prove, in the first place, by use of the axioms 2,3 and $5_{1}$, that the

* That in an $n$-mpace there exists no set of $n+2$ independent points and thus no $(n+1)$-space is a theorem. (Cf. § 3.) 
statements are consequences* of the single statement $2_{1}$, which, in modified notation, is :

$$
\text { 2. If } A B C \text { and } A C D \text {, then } B C D \text {. }
$$

One observes readily that statements 3 and 4 follow from statements 1 and 2 . Now statement $2_{2}$ follows from $2_{1}$, for we have $C A B$ and $A D B$, and if we had $D B C$, then by $2_{1}$ from $D B C$ and $B A C$ we should have $D B A$ in contradiction with $A D B$; and if we had $B C D$, then by $2_{1}$ from $D C B$ and $C A B$ we should have $D C A$, and, at the same time, from $A D B$ and $D C B$ we should have $A D C$; these two conclusions are contradictory; hence we have, as stated, $C D B$.

Next the statements $1_{1}$ and $1_{2}$ are evidently equivalent, and we prove the truth of $1_{1}$ on the assumption that $2_{1}$ and consequently $2_{2}$ are valid.

Changing the notation, we have $A B C$ and $B C D$ and are to prove $A B D$. If we had $A D B$, then by $2_{1}$ from $C B A$ and $B D A$ we should have $C B D$, which is impossible. We must prove that $D A B$ is impossible. Suppose we had $D A B$ with $A B C$ and $B C D$. If we had $A C D$, then by $2_{2}$ from $D C A$ and $C B A$ we should have $D B A$, which is impossible. If we had $D A C$, then by $2_{2}$ from $D A C$ and $A B C$ we should have $D B C$, which is impossible. Hence we should have $C D A$, and so the four points $A, B, C, D$ would have the relations $A B C, B C D, C D A, D A B$; this set of four relations we denote by the notation $(A B C D)$ cyclic.

Accordingly we have at least proved that if $A B C$ and $B C D$, then either $A B D$ or $(A B C D)$ cyclic, and so likewise either $D C A$ or $(D C B A)$ cyclic ; that is, if $A B C$ and $B C D$, then either $A B D$ and $A C D$ or else $(A B C D)$ cyclic.

We consider the case $(A B C D)$ cyclic. By axiom $5_{1}$ there is in this case on the line $A B C D$ a point $O$ different from $A, B, C, D$ and such that $D O A$. By 2 from $C D A, D O A, D A B$ we have $C D O, C O A, D O B, O A B$.

Further, from $B C D$ and $C D O$ we have, in accordance with the alternative above proved, either $B C O$ and $B D O$ or else $(B C D O)$ cyclic. The former of these alternatives contradicts the result $D O B$ obtained previously. Thus we have $(B C D O)$ cyclic, and from it $D O B, O B C$.

Similarly, from $O A B$ and $A B C$ we have either $O A C$ and $O B C$ or else $(O A B C)$ cyclic. The latter of these alternatives is contradictory to the result $O B C$ just obtained, while the former is contradictory to the result $C O A$ obtained earlier. Thus, by the intervention of the point $O$, we recognize that the case $(A B C D)$ cyclic is impossible. Hence 1 follows from $2_{1}$.

Accordingly we have proved that the four statements 1-4 depend, by use of the axioms 2,3 and $5_{1}$, upon the single statement $2_{1}$ :

* Mr. VeBLen has found that HILBert's axiom II 4 is deducible from the two statements :If $A B C$ and $A C D$, then $A B D$. If $A B D$ and $A C D$, then $A B C$ or $C B D$. 


\section{If $A B C$ and $A C D$, then $B C D$.}

We are to prove further, by the use of axioms 1,6 in addition to those previously mentioned, that this statement 2 depends upon axiom 4 , the triangletransversal axiom.

This will be proved in three parts:

(a) The relations $A B C, A C D, C D B$ cannot hold simultaneously.

( $\beta$ ) If $A B C$ and $A C D$, then $A B D$.

$(\gamma)$ The relations $A B C, A C D, D B C$ cannot hold simultaneously.

Here $(\beta)$ depends upon $(\alpha)$ and is a lemma for $(\gamma)$, while $(a)$ and $(\gamma)$ together yield the theorem in question.

In proof of $(a):$ We take a point $E$, not on the line $A B C D$; we draw the distinct lines $E C, E B$; and on the segment $E C$ we select a point $F$. Then, in accordance with axiom 4 and its first corollary as applied to the transversal $A F$ of the triangle $E B C$, in view of the relations $A B C$ and $E F C$, we see that the line $A F^{\prime}$ cuts the segment $E B$ in a point $G$ for which $E G B$ and $A G F$. In the triangle $E B C$ the segment $F D$ cutting the two segments $E C, B C$ (in view of the relation $C D B$ ) is itself cut by the line $C G$, in accordance with cor. 5 of axiom 4, in a point $H$ for which $F H D$. Then (in view of the relation $A C D$ ) the line $C G H$ cuts the three sides of the triangle $A D F$ in three inner points. But this, by cor. 7 of axiom 4, is impossible. Accordingly statement $(\boldsymbol{a})$ is proved.

In proof of $(\beta)$ : The case $A B C, A C D, B A D$ (that is, $D C A, I) A B$, $A B C)$ is excluded by $(a)$, and the case $A B C, A C D, A D B$ leads by $(a)$ to this impossibility that no one of the three points $B, C, D$ is between the other two. Accordingly statement $(\beta)$ is proved.

In proof of $(\gamma)$ : We take a point $E$, not on the line $A B C D$; we draw the line $E C$; and on the segment $E C$ we select a point $F$. Then, in view of $E F C$ and $A C D$, the transversal $A F$ cuts the side $E D$ of the triangle $E C D$ in a point $G$, where $E G D$ and $A F G$.-Further, in view of $A B C$ and $A F G$, the transversal $G B$ of the triangle $A C F$ cuts the segment $C F$ in a point $H$; for this point $H$, in view of $C H F$ and $C F E$, we have by $(\beta)$ the relation $C H E$. Then the transversal $B G H$, in view of $D B C, D G E$ and $C H E$, meets the three sides of the triangle $D C E$ internally, and this is impossible.

\section{§3. Properties of the k-spaces of the fundamental space.}

We suppose that axioms 1-6 with $n \geqq 2$ hold for the fundamental space under consideration in $\S 3$. By the first statement of the following theorem it appears that a $k$-space of the fundamental space is a fundamental $k$-space in the sense of $\S 1$, the axioms 1-7 holding with $k$ as $n$; those points, lines, segments of the fundamental space, which lie in the $k$-space, being directly identified with the points, lines, segments of the fundamental $k$-space. 
Theorem.-In a fundamental space for which the axioms 1-6 with $n \geqq 2$ are valid, the sets of independent points and the $k$-spaces have the following properties:

1. Any two distinct points of a $k$-space determine a line or 1-space lying entirely in it.

2. A k-space is determined by any set of $k+1$ independent points lying in it.

3. A $k$-space contains no set of $k+2$ independent points.

4. Any $k^{\prime}+1$ independent points of a $k$-space $\left(0 \leqq k^{\prime} \leqq k\right)$ determine a $k^{\prime}$-space lying entirely in it.

5. If $k+1$ points are independent when taken in a certain order, they are independent when taken in any order.

6. $A(k-1)$-space lying in a k-space separates the points of the $k$-space which do not lie in the $(k-1)$-space into two sets or parts in such a way that two points of the same part are not separated by the $(k-1)$-space.

7. In a k-space, a p-space and a q-space $(p+q \geqq k)$ having a common point have in common precisely an r-space, where $r$ is a definite integer such that $r \geqq p+q-k$ and (by 3$) 0 \leqq r \leqq p, 0 \leqq r \leqq q$.

In view of the theorem of $\S 2$ this theorem is true for the case $k=1$, a 0 -space being a point. Its truth for the general $k$ may be proved by the induction from $k$ to $k+1$. The general step of induction will however be clearer if the proof for the case $k=2$ is given by itself.

For the case $k=2$ we consider three independent points $A_{1}, A_{2}, B_{0}$. We denote by $a$ the line $A_{1} A_{2}$; by $\{A\}$ the set of points $A$ of the line $a$; by $\{C\}$ the set of points $C$ separated from $B_{0}$ by $a$; with respect to a particular point $C$, by $\left\{B_{C}\right\}$ the set of points $B_{C}$ separated from the point $C$ by $a$; by $\left\{\left\{B_{C}\right\}\right\}$ the aggregate of all such sets $\left\{B_{C}\right\}$. In these notations the plane $\pi=A_{1} A_{2} B_{0}$ is by def. 2 of $\S 1$ a certain set $\pi=\{P\}$ of points $P$, viz:

$$
\{P\}=\{A\}+\{C\}+\left\{\left\{B_{C}\right\}\right\} \text {. }
$$

The proof of the statements of the theorem may easily be made to depend on the following three remarks :

The plane $\pi=A_{1} A_{2} B_{0}=a B_{0}$ contains all the lines of the type $A B_{0}$.

The two planes $\pi=A_{1} A_{2} B_{0}$ and $\pi^{\prime}=A_{1} A_{2} B_{0}^{\prime}$, where $B_{0}^{\prime}$ is any point $P$ of $\pi$ not in $a$, are identical.

The two planes $\pi=A_{1} A_{2} B_{0}$ and $\pi^{\prime}=A_{1} B_{0} A_{2}$ are identical.

In proof of the first remark the theorem of $\S 2$ is at once applicable.

In proof of the second remark we have in mind axioms 4 and 5 and the theorem of $\S 2$. In the plane $\pi=A_{1} A_{2} B_{0}=a B_{0}$, the line $a$ does not separate two points $C$. Further the sets $\left\{B_{C_{1}}\right\},\left\{B_{C_{2}}\right\}$ connected with two distinct points $C_{1}, C_{2}$ of the set $\left\{C_{\}}\right\}$are identical; this is true if the line $C_{1} C_{2}$ contains a 
point $A$, and likewise, if the line $C_{1} C_{2}$ contains the point $B_{0}$, and further if the two lines $B_{0} C_{1}, B_{0} C_{2}$ are distinct, in view of the fact that on the line $B_{0} C_{2}$ there is a point $C_{3}$ of the set $\{C\}$ such that the line $C_{1} C_{3}$ contains a point $A$. Thus the various sets $\left\{B_{c}\right\}$ are identical with one another; we set $\left\{B_{C}\right\}=\{B\}$ and have

$$
\{\boldsymbol{P}\}=\{\boldsymbol{A}\}+\left\{\boldsymbol{C}^{\prime}\right\}+\{\boldsymbol{B}\}=\{\boldsymbol{A}\}+\{\boldsymbol{B}\}+\{\boldsymbol{C}\} .
$$

The sets $\{B\},\{C\}$ have no points in common, since two points $C_{1}, C_{2}$ or $B_{1}, B_{2}$ are not separated by $a$, while two points $B, C$ are separated by $a$. The line $a$ separates the plane $\pi$ into the two parts $\{B\},\{C\}$. It is now easy to see that the plane $\pi=A_{1} A_{2} B_{0}$ is identical with the plane $\pi^{\prime}=A_{1} A_{2} B_{0}^{\prime}$, if $B_{0}^{\prime}$ is any particular point $C$, and so, by repetition of the argument, also if $B_{0}^{\prime}$ is any particular point $B$. Accordingly the second remark is proved.

From the second remark a point $P$ of the plane $\pi=A_{1} A_{2} B_{0}=a B_{0}$ is a point $A$ of the line $a=A_{1} A_{2}$, or a point $A^{\prime}$ of line $a^{\prime}=A_{1} B_{0}$, or a point $P$ such that for certain two distinct points $A_{3}$ of $a, A_{3}^{\prime}$ of $a^{\prime}$ the relation $A_{3}^{\prime} A_{3} P$ holds ; in the last case selecting a point $A_{4}$ of $a$ such that $A_{3} A_{1} A_{4}$, we find a point $A_{4}^{\prime}$ of $a^{\prime}$ such that $A_{4} A_{4}^{\prime} P$. Accordingly the planes $A_{1} A_{2} B_{0}$ and $A_{1} B_{0} A_{2}$ are identical.

We proceed to prove the truth of the theorem in question for the case $k=m+1$ on the assumption of its validity for the case $k=m$.

We consider the $(m+1)$-space $\Sigma=A_{1} A_{2} \cdots A_{m} A_{m+1} B_{0}=a B_{0}$. One proves as before (but with the use of statement 1 of the theorem for $k=m$ ) that, in notations analogous to those previously used,

$$
\Sigma=\{S\}=\{A\}+\{B\}+\{C\},
$$

and that the $(m+1)$-space $\Sigma=a B_{0}$ is identical with the $(m+1)$-space $\Sigma^{\prime}=a B_{0}^{\prime}$, where $B_{0}^{\prime}$ is any point of $\Sigma$ not in $a$.

Further, one sees that the $(m+1)$-space $\cdot \Sigma$ contains the $m$-space $a$, that all lines joining points $S$ to points $A$ lie in $\Sigma$, and that the $m$-space $a$ divides the space $\Sigma$ into two parts as specified in statement 6 .

By statement 2 for the $m$-spaces, the space $\Sigma$ may then be obtained from any $m+1$ independent points of $a$ together with any point $S$ of $\Sigma$ not in $a$.

Statements 1 to 6 will follow easily from the remarks already made, when we prove that the two* $(m+1)$-spaces,

$$
\Sigma=A_{1} \cdots A_{m} A_{m+1} B_{0}, \quad \Sigma^{\prime}=A_{1} \cdots A_{m} B_{0} A_{m+1}
$$

are identical, and in proof of this last statement it is convenient to modify the notations. The $(m-1)$-space $A_{1} \cdots A_{m}$ is denoted by $d=D_{1} \cdots D_{m}$; the

\footnotetext{
* One uses statement 3 for $k=m$.
} 
points $A_{m+1}, B_{0}$ are denoted by $A_{0}, A_{0}^{\prime}$; and the lines $D_{m} A_{0}, D_{m} A_{0}^{\prime}$ are denoted by $l, l^{\prime}$. We have then

$$
\begin{array}{ll}
a=d A_{0}=d l, & a^{\prime}=d A_{0}^{\prime}=d l^{\prime}, \\
\Sigma=a A_{0}^{\prime}=a l^{\prime}, & \Sigma^{\prime}=a^{\prime} A_{0}=a^{\prime} l .
\end{array}
$$

We see that the statement is proved when we prove that every point $S$ of $\Sigma$ is a point $S^{\prime}$ of $\Sigma^{\prime}$. This is true evidently for a point $S$ which is a point $A$ of $a$ or a point $A^{\prime}$ of $a^{\prime}$ or, by the discussion of the third remark for $k=2$, a point of the plane $D_{n} A_{0} A_{0}^{\prime}=D_{n} A_{0}^{\prime} A_{0}=l l^{\prime}=l^{\prime} l$. - We consider any other point $S$ of $\Sigma$. Such a point $S$ is collinear with certain points $A$ of $a$ and $L^{\prime}$ of $l^{\prime}$, where $L^{\prime} A S$; and in its turn $A$, lying in $a=d l$ and lying neither in $d$ nor in $l$, is collinear with certain points $D$ of $d$ and $L$ of $l$, where $L D A$; and accordingly, by axiom $4, S$ is collinear with the point $L$ and a point $A^{\prime}$ of the segment $L^{\prime} D$ of the space $a=d l^{\prime}$; this relation $L A^{\prime} S$ indicates that the point $S$ is a point $S^{\prime}$ of $\Sigma^{\prime}=a^{\prime} l$. - This completes the proof of statements 1 to 6 for the general $k$.

It remains to prove the validity of statement 7 . We suppose that in the $k$-space $\Sigma$ a $p$-space $a$ and a $q$-space $\beta(p+q \geqq k)$ have a common point $C_{0}$. Then they have in common precisely an $r$-space $\gamma$, where $0 \leqq r \leqq p, 0 \leqq r \leqq q$. We are to show that $r \geqq p+q-k$.

We take $\gamma$ as determined by the $r+1$ linearly independent points $C_{0}, \ldots, C_{r}$; and $a$ as determined by these points with $p-r$ points $A_{1}, \cdots, A_{p-r}$; and $\beta$ as determined by these points with $q-r$ points $B_{1}, \ldots, B_{q-r}$. If $\beta$ lies in $a$, then $r=q$; hence, since $k \geqq p$, we have indeed $r \geqq p+q-k$.

We suppose then that $\beta$ does not lie in $a$; thus there is a point $B_{1}$. Then the $(p+1)$-space $a B_{1}$ has in common with the $q$-space $\beta$ precisely the $(r+1)$-space $\gamma B_{1}$. For let $B^{\prime}$ be any point common to $a B_{1}$ and $\beta$. If $B^{\prime}$ is of $a$, then it is of $\gamma$ and thus of $\gamma B_{1}$. If it is not of $a$, then in the $(p+1)$-space $a B_{1}$ it lies either on the opposite side of $a$ from $B_{1}$, in which case the segment $B_{1} B^{\prime}$ contains a point $A$ of $a$ which is, however, likewise a point $B$, and thus is a point $C$ of $\gamma$, and thus $B^{\prime}$ is of the line $C B_{1}$ and hence of the $(r+1)$-space $\gamma B_{1}$; or it is on the same side of $a$ as $B_{1}$, in which case a similar result will follow by the mediation of a point $B_{1}^{\prime}$ lying on the line through $B_{1}$ and the point $C_{0}$ and on the opposite side of $a$ from the point $B_{1}$. So we see that if the spaces $a$ and $\beta$ have $\gamma$ in common, then the spaces $a B_{1}$ and $\beta$ have precisely $\gamma B_{1}$ in common. Hence $a B_{1}$ does not contain $B_{2}$. Similarly, $a B_{1} B_{2}$ and $\beta$ have precisely $\gamma B_{1} B_{2}$ in common, and $a B_{1} B_{2}$ does not contain $B_{3}$. Thus it appears finally that the points $C_{0}, \cdots, C_{r}, A_{1}, \cdots, A_{p-r}, B_{1}, \cdots, B_{q-r}$ are $1+p+q-r$ independent points of the $k$-space $\Sigma$, and accordingly we have $p+q-r \leqq k$, that is, $r \geqq p+q-k$, which was to be proved. 
§4. The figure in a k-space determined by $k+1$ independent points.

In a $k$-space a $(k+1)$-gon $A_{1} \cdots A_{k+1}$ whose $k+1$ vertices $A_{g}$ are independent points determines by its $k+1(k-1)$-space faces $a_{1} \ldots a_{k+1}$ a certain partition of the $k$-space.

To every point $P$ of the $k$-space there is assigned a certain $(k+1)$-partite character : every partial character of $P$ is,+ 0 , or - ; the $g$ th partial character is 0 if $P$ lies in $a_{g}, \pm$ if $P$ and $A_{g}$ lie on the same side $\begin{gathered}\text { the } \\ \text { op posite sides }\end{gathered} a_{g}$. Thus, for instance, $A_{1}$ has the character $(+, 0, \ldots 0)_{k 0^{\prime},} \cdot$ From the $g$ th partial characters of two points $P_{1}, P_{2}$ one infers at once whether the segment $P_{1} P_{2}$ has lying in $a_{g}$ no point, one point, or all its points.

Points having the same character are classed together and form one of the parts in the partition in question. There are $3^{k+1}$ characters; of these, however, only those containing at least one partial character + are characters of points. This is provable by induction. One notes, for instance, that the $(k+1)$-partite characters of points of the $k$-space $a_{k+1}=A_{1} \cdots A_{k}$ are the $k$-partite characters of these points as points of $a_{k+1}$ with the adjunction of 0 as $(k+1)$ th character.

The points with character $(+, \cdots,+)_{k+1}+{ }^{\prime}$ constitute the interior $*$ of the $(k+1)$-gon, while similarly the points with no partial character - constitute, as variously grouped, the interiors of the various faces of various dimensions of the $(k+1)$-gon. If $P$ is a point of the interior of the $(k+1)$-gon, the line $A_{g} P$ cuts the interior of the face $a_{g}$ in a point $A_{g}^{\prime}$ such that $P$ lies between $A_{g}$ and $A_{g}^{\prime}$.

We speak of compleinentary or opposite faces of the $(k+1)$-gon. $\dagger$ If a point $P$ lies on no face and also not in the interior of the $(k+1)$-gon, then (in only one way) there are two points $Q, R$ lying in the interior of opposite faces of the $(k+1)$-gon and such that $Q$ lies on the segment $P R$. The character of $P$ is made up of say $q$ +'s and $r$-'s $(q+r=k+1 ; q>0, r>0)$, and, by convenient determination of the notation, it is $(+, \cdots,+,-, \cdots,-)_{q_{+}{ }_{s} ; r_{-}^{\prime} s}$; then the points $Q, R$ have respectively the characters

$$
(+, \cdots,+, 0, \ldots, 0)_{q+{ }^{\prime} s ; r 0^{\prime} s}, \quad(0, \ldots, 0,+, \ldots,+)_{q 0^{\prime} s ; r+{ }^{\prime}} .
$$

The definition of the $k$-space analogous to the Peano-Ingrami definition $\ddagger$ of the plane and of the 3 -space is the following:

$\mathrm{A}(k+1)$-gon $A_{1} \cdots A_{k+1}$ lying in no $(k-1)$-space determines as its interior the set of all points lying on the segments connecting its vertices to points lying in the interior of the respectively opposite $k$-gon faces; and it determines as the $k$-space $A_{1} \cdots A_{k+1}$ the set of all points lying either on one of the $(k-1)$-space

* For $k=0$ : a point constitutes simultaneously a 0 -space, a 1 -gon, the interior of a 1-gon.

$\dagger$ For example : $(k=2)$ in a triangle a vertex and opposite side are complementary faces ; $(k=3)$ in a tetrahedron a vertex and opposite face are complementary faces, and two opposite edges are complementary faces.

$\ddagger$ Cf. Schur, loc. cit., p. 268. 
faces of the $(k+1)$-gon or on a line intersecting the interiors of two opposite faces of the $(k+1)$-gon.

This definition and that of $\S 1$ are equivalent. Although in this definition the $k+1$ determining points enter symmetrically, the definition of $\S 1$ is apparently much simpler.

\section{§5. The Hilbert plane.}

We consider a Hilbert geometry in which the basal elements are points, lines, segments of lines and Hilbert planes subject to the Hilbert axioms I 1, 2, $7_{1}$, II 1, 2, 3, which do not involve the Hilbert plane, and furthermore subject to the following axioms I $7_{3}^{\prime}, 7_{2}^{\prime}, 3^{\prime}, 5^{\prime}$, II $5^{\prime}$, which do involve and furnish the implicit definition of the Hilbert plane. These axioms I $7_{3}^{\prime}, 7_{2}^{\prime}, 3^{\prime}, 5^{\prime}$, II $5^{\prime}$ are (by use of I 1, 2, $7_{1}$, II $1,2,3$ ) deducible from the Hilbert axioms $\mathrm{I}_{3}$, $7_{2}, 3,5$, II 5 .

I $7_{3}^{\prime}, 7_{2}^{\prime}$. There exists a Hilbert plane. Every Hilbert plane $\pi$ has a notation $A B C$ with respect to certain $*$ three non-collinear points $A, B, C$. In the notation $A B C$ there is no question of order of the three points $A, B, C$.

I 3 '. Three non-collinear points $A, B, C$ determine ${ }^{*}$ a set of points: a Hilbert plane $A B C$, to which they belong.

I 5'. With respect to a Hilbert plane $A B C$ every line joining any point $A^{\prime}$ of the segment $B C$ to a point $D$ distinct from $A^{\prime}$ and lying in the plane lies wholly in the plane.

II 5'. (The plane order axiom). With respect to a Hilbert plane $A B C$ every line which lies in the plane and which contains a point of the segment $B C$ contains also $A$ or $B$ or $C$ or a point of the segment $C A$ or a point of the segment $A B$.

In this geometry we prove first the validity, as theorems, of the axioms 1-5 and $6(n=2)$ of $\S 1$; from them follow by $\S 2$ the validity as a theorem of the Hilbert axiom II 4 , by $\S \S 3,4$ the theory of the plane as defined in $\S 1$, and the identity of this plane with that defined by Peano.

Now we consider any Hilbert plane $A B C$ with notation connected with three non-collinear points $A, B, C$. It contains the points $A, B$ and $C$, the line $B C$ (in view of the existence of a point $A^{\prime}$ of the segment $B C$ ), the lines $C A$ and $A B$, and all lines of the types $A A^{\prime}, B B^{\prime}, C C^{\prime}$ where $A^{\prime}, B^{\prime}, C^{\prime}$ are points of the respective segments $B C, C A, A B$. Hence the Hilbert plane $A B C$ contains the Peano plane $A B C$. Further, if $D$ is a point of the Hilbert plane $A B C$ not on one of the lines $B C, C A, A B$, then the line $A^{\prime} D$ (where $A^{\prime}$ is a point of the segment $B C$ ) contains the point $A$ or a point $B^{\prime}$ of the segment $C A$ or a point $C^{\prime}$ of the segment $A B$. The line $A^{\prime} D$ quâ $A^{\prime} A$ or $A^{\prime} B^{\prime}$ or $A^{\prime} C^{\prime}$

* It is not implied that a plane $\pi$ has such notation $A B C$ in a definite way, nor that a notation $A B C$ denotes a definite plane. 
lies entirely in the plane $A B C$ (of $\S 1)$. Hence this plane $A B C$ contains the Hilbert plane $A B C$.

Accordingly the plane $A B C$ (of $\S 1$ ), the Peano plane $A B C$ and the Hilbert plane $A B C$ are identical. Then by $\S 3$ follows the validity as theorems for the Hilbert plane of the Hilbert axioms I $3,4,5,7_{2}$, II 5 in their fulness.

Hence the Hilbert axioms I 4, II 4 are logically deducible from the Hilbert axioms I 1-3, 5, 7, II 1-3, 5.-If we had wished to exhibit merely this dependence it would have been simpler not to introduce the axioms $I 7_{2}^{\prime}, 7_{3}^{\prime}, 3^{\prime}$, $5^{\prime}$, II $5^{\prime}$

The University of Chicago, December 31, 1901. 